\title{
Cost-effectiveness analysis comparing ceftazidime/avibactam (CAZ-AVI) as empirical treatment comparing to ceftolozane/tazobactam and to meropenem for complicated intra- abdominal infection (cIAl)
}

Thitima Kongnakorn ${ }^{*}$, Christian Eckmann ${ }^{2}$, Matteo Bassetti ${ }^{3}$, Eszter Tichy ${ }^{4}$, Roberto Di Virgilio ${ }^{5}$, Nathalie Baillon-Plot ${ }^{6}$ and Claudie Charbonneau ${ }^{6}$

\begin{abstract}
Background: The rising incidence of resistance to currently available antibiotics among pathogens, particularly Gram-negative pathogens, in complicated intra-abdominal infections (cIAls) has become a challenge for clinicians. Ceftazidime/avibactam (CAZ-AVI) is a fixed-dose antibiotic approved in Europe and the United States for treating (in combination with metronidazole) $\mathrm{CIAI}$ in adult hospitalised patients who have limited or no alternative treatment options. The approval was based on the results of RECLAIM, a Phase III, parallel-group, comparative study (RECLAIM 1 [NCT01499290] and RECLAIM 2 [NCT01500239]). The objective of our study was to assess the cost-effectiveness of CAZ-AVI plus metronidazole compared with 1) ceftolozane/tazobactam plus metronidazole and 2) meropenem, as an empiric treatment for the management of CIAl in Italy.

Methods: A sequential, patient-level simulation model, with a 5-year time horizon and 3\% annual discount rate (applied to both costs and health benefits), was developed using Microsoft Excel ${ }^{\varpi}$ to demonstrate the clinical course of the disease. The impact of resistant pathogens was included as an additional factor.

Results: In the base-case analysis, the CAZ-AVI sequence (CAZ-AVI plus metronidazole followed by a colistin + tigecycline + high-dose meropenem combination after treatment failure), when compared to sequences for ceftolozane/tazobactam (ceftolozane/tazobactam plus metronidazole followed by colistin + tigecycline + high-dose meropenem after treatment failure) and meropenem (meropenem followed by colistin + tigecycline + high-dose meropenem after treatment failure), had better clinical outcomes with higher cure rates (93.04\% vs. 91.52\%; 92.98\% vs. $90.24 \%$, respectively), shorter hospital stays ( $\Delta=-0.38$ and $\Delta=-1.24$ days per patient, respectively), and higher quality-adjusted life years (QALYs) gained per patient (4.021 vs. 3.982; 4.019 vs. 3.960, respectively). The incremental cost effectiveness ratio in the CAZ-AVI sequence was $€ 4099$ and $€ 15,574$ per QALY gained versus each comparator sequence, respectively, well below the willingness-to-pay threshold of $€ 30,000$ per QALY accepted in Italy.

(Continued on next page)
\end{abstract}

\footnotetext{
* Correspondence: thitima.kongnakorn@evidera.com

'Evidera, The Ark, 201 Talgarth Road, Hammersmith, London W6 8BJ, UK

Full list of author information is available at the end of the article
}

(c) The Author(s). 2019 Open Access This article is distributed under the terms of the Creative Commons Attribution 4.0 International License (http://creativecommons.org/licenses/by/4.0/), which permits unrestricted use, distribution, and reproduction in any medium, provided you give appropriate credit to the original author(s) and the source, provide a link to the Creative Commons license, and indicate if changes were made. The Creative Commons Public Domain Dedication waiver (http://creativecommons.org/publicdomain/zero/1.0/) applies to the data made available in this article, unless otherwise stated. 
(Continued from previous page)

Conclusions: The model results demonstrated that CAZ-AVI plus metronidazole could be a cost-effective alternative when compared with other antibiotic treatment options, as it is expected to provide better clinical benefits in hospitalised patients with clAI in Italy.

Keywords: Economic model, Cost-effectiveness analysis, Ceftazidime-avibactam plus metronidazole, Ceftolozane/ tazobactam plus metronidazole, Meropenem, Complicated intra-abdominal infection

\section{Background}

Complicated intra-abdominal infections (cIAI) arise due to perforation or necrosis of the gastrointestinal tract viscera [1]. Invasion of peritoneal and retroperitoneal space by bacteria further results in localized or diffuse peritonitis $[2,3]$. According to a study by World Society of Emergency Surgery, the overall mortality rate was $10.5 \%$ [4]. Several Gram-negative bacteria, including Escherichia coli, Klebsiella pneumoniae, and the Enterobacter species, as well as other resistant pathogens, have been implicated in cIAIs and are believed to be accountable for more than $70 \%$ of the cases reported worldwide [5]. According to the United States (US) Centers for Disease Control and Prevention, some of the major Gram-negative bacteria that cause cIAIs and similar infections have developed resistance to currently available antibiotic drugs [6]. As bacterial resistance has increased, both the human and economic costs of treating resistant infections have risen, global concern has escalated, and the need to develop newer antibacterial agents has intensified [7].

Ceftazidime/avibactam (CAZ-AVI) is a novel, $\beta$ lactam/ $\beta$-lactamase inhibitor fixed-dose combination drug containing ceftazidime (an established, extendedspectrum and avibactam (a unique, non- $\beta$-lactam, $\beta$ lactamase inhibitor) $[1,8]$. CAZ-AVI has been approved by the European Commission and the US Food and Drug Administration (FDA) for the indication of cIAI (in combination with metronidazole) in adult patients $[1,9]$. The introduction of CAZ-AVI is encouraging as this novel combination drug is known to have potent activity against several Gram-negative organisms, including some with multidrug resistance [9]. Hence, it could be a viable answer to rising incidence of resistance to most of the currently available antibiotic treatments that has not only become a challenge for clinicians to treat the disease but also has increased hospital stays as well as soaring healthcare costs related to cIAIs $[3,7]$.

Two identical, prospective studies (RECLAIM 1 [NCT01499290] and RECLAIM 2 [NCT01500239]) were conducted to evaluate the efficacy and safety of CAZAVI plus metronidazole compared to meropenem, followed by appropriate intravenous infusion, in hospitalised patients with cIAI. With the agreement of the US FDA and the European Medicines Agency (EMA), data from both these studies were combined to form a single inferential database [1].

Clinical cure at the test-of-cure (TOC) visit was the primary endpoint to assess the non-inferiority of CAZAVI plus metronidazole vs. meropenem. The assessment was performed in the microbiologically modified intention-to-treat population, and the modified intention-to-treat and clinically evaluable populations in the US and Europe, as requested by the FDA and EMA, respectively. RECLAIM was designed as a non-inferiority study. Meropenem, the current best practice drug for cIAIs, was selected as the comparator of CAZ-AVI.

In 2001, the Italian Group for Pharmacoeconomic Studies published guidelines for economic evaluations; these specify that cost-effectiveness assessments of newer agents in comparison to drugs already in the market should include the perspective of both healthcare providers and payers [10]. Economic evaluations (cost effectiveness and cost utility) are particularly important with new antibiotics such as CAZ-AVI, helping to establish their true market value when the interpretation of clinical trial results is limited by the increasing incidence of resistant pathogens [11, 12].

Italy is a top country in Europe with high prevalence of resistant Gram-negative pathogens. In 2014, K. pneumoniae isolates were resistant to carbapenems in approximately 25-50\% of the cases; $P$. aeruginosa isolates were resistant to carbapenems in $25-50 \%$ of the cases and up to $10-50 \%$ of strains were classified as multi-drug resistant; $A$. baumannii had combine resistance to fluoroquinolones, aminoglycosides, and carbapenems in up to $50 \%$ of cases [13]. Furthermore, the antibiotics consumption out of hospitals was 27.8 doses per 1000 inhabitants, ranking the fifth country with high use of antibiotics in Europe [14].

The objective of this study was to analyse the cost effectiveness of CAZ-AVI plus metronidazole as an empiric treatment compared with meropenem or with ceftolozane/tazobactam plus metronidazole for appropriate hospitalised adult patients with cIAI from the Italian publicly funded healthcare (third-party payer) perspective.

\section{Methods}

Model structure

A sequential, patient-level simulation model was developed in Microsoft Excel $^{\odot}$ to simulate the clinical course of cIAI, from diagnosis until clinical cure or death, after 
the initiation of empiric therapy. An overview of the model structure describing each patient's pathway is shown in Fig. 1.

To begin with, 5000 patients hospitalised for cIAI were created in the model and every patient was assigned clinical characteristics using Monte Carlo sampling for type and resistance of infecting pathogen(s). These simulated patients were then duplicated in the model to create two identical sets of patients, to ensure that no factor other than the treatment had any influence on the outcome. The patients in one set received CAZ-AVI plus metronidazole as their empiric treatment, while those in the other received either meropenem, a widely used antibiotic for cIAI, or ceftolozane/tazobactam plus metronidazole, one of the newly approved antibiotics for cIAI at the time of this study.

Each patient entered the model on empiric treatment and the treatment was continued for the next $48-72 \mathrm{~h}$ (i.e., until microbiological results were available). If no resistance was observed in a patient's test results, then the empiric treatment was continued (i.e., appropriate empiric treatment). Any patient whose test showed at least one pathogen resistant to the empiric treatment was then switched to the next treatment line and was counted as a clinical failure (Fig. 1).

At the end-of-treatment (EOT) visit, patients were assessed for response. If a response was achieved (i.e., appropriate empiric treatment), then the patient was subsequently assigned for assessment at the first follow- up visit (i.e., 28-35 days following initiation of treatment), equivalent to a TOC visit during a clinical trial. If no response was achieved (i.e., inappropriate empiric treatment), the patient was moved to the next treatment line and was counted as a clinical failure.

At the first follow-up visit, patients were assessed for clinical cure. If clinical cure was achieved, then the patient was subsequently assigned for assessment at the second follow-up visit (i.e., 42-49 days post-treatment initiation), equivalent to a long-term follow-up (LFU) visit in the clinical study. If clinical cure was not observed, the patient was switched to the next treatment line.

At the second follow-up visit, patients were assessed for recurrence of infection (equivalent to clinical failure observed at LFU visit in a randomized controlled study). If a patient had a recurrence, a one-time cost of recurrence was accrued; that cost was assumed to include all medical expenditures pertaining to management of the recurrence, such as cost of medication (e.g., antibiotics) and hospitalisation.

During the simulation of the clinical course, each patient was also exposed to risks of treatment-related adverse events (AEs) and in-hospital death. When an $\mathrm{AE}$ occurred, the medical cost of managing the $\mathrm{AE}$ was accrued and some patients may discontinue the AE-related treatment and switch to the next treatment or to best supportive care. All cost outcomes, life years (LYs) and quality-adjusted life years (QALYs) were accrued over the time horizon or until the patients died.

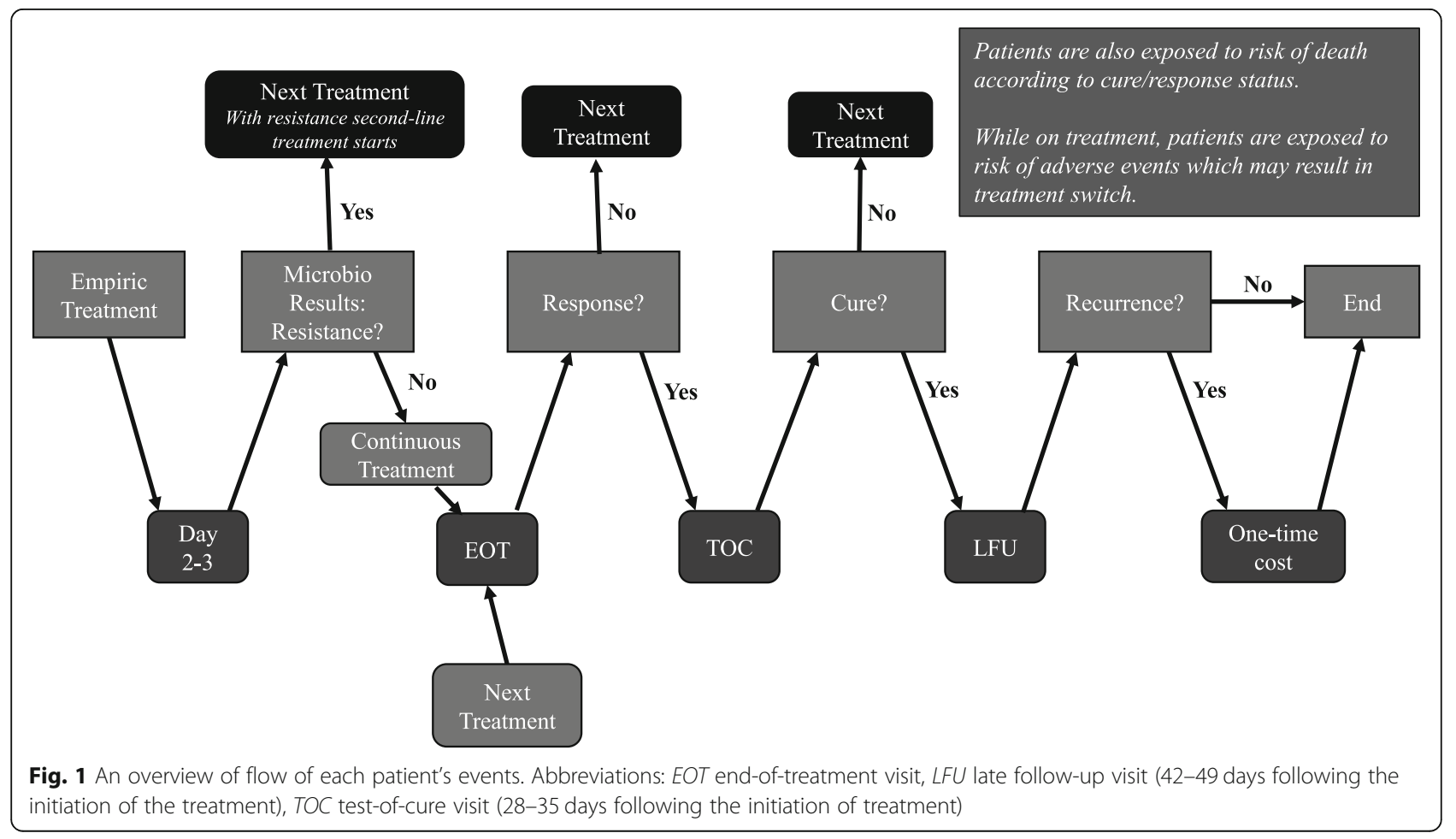


The model allowed inputs on resistance of pathogens to have impact on model outcomes. To incorporate the additional burden of antibiotic resistance (which cannot typically be captured in clinical trials), three assumptions were made in the base case. First, a 10\% additional cost of hospitalisation was assumed to cover the increased utilization of resources to treat patients with antibioticresistant pathogens [14]. Next, the mortality rate for patients with antibiotic-resistant disease was assumed to be $20 \%$ higher compared to those patients who had no resistance but received inappropriate antibiotic therapy [14-16]. Last, based on clinical expert opinion, the efficacy of subsequent treatment was assumed to be decreased by $10 \%$, as resistance to the empiric treatment may have impact on the subsequent treatments. All these three assumptions were tested in a scenario analysis.

\section{Treatment comparison}

In the model, each sequence was composed of an empiric treatment followed by a second line of treatment, that was administered after the failure of the empiric treatment for any reason (e.g., resistance, lack of response or clinical cure, serious adverse event [SAE]). The treatment sequences were in accord with current treatment strategies in Italian clinical practice, based on clinical advice. Although some publications suggest that the monotherapy of colistin is not inferior to colistin combination therapy [17-19], these papers evaluate more broader spectrum of disease or other disease as our current evaluations and the data these publications use are not Italy-specific. Therefore, we relied on the opinion of clinical experts for the second-line treatment in our evaluation and a triple combination therapy of colistin + tigecycline + high-dose meropenem was selected. A scenario analysis was run using cost of colistin monotherapy instead of the triple combination in second-line, the results showed small increase in the incremental cost-effectiveness ratios (ICERs), they still remained below the threshold of $€ 30,000$ per QALY. There were a total of three treatment sequences: 1) empiric treatment with CAZ-AVI plus metronidazole, followed by a second-line treatment of a combination of colistin (intravenous) and tigecycline plus high-dose meropenem (colistin + tigecycline + high-dose meropenem), called the 'CAZ-AVI sequence'; 2) empiric treatment with ceftolozane/tazobactam plus metronidazole, followed by colistin + tigecycline + high-dose meropenem (the 'ceftolozane/tazobactam sequence'); and 3) meropenem alone, followed by colistin + tigecycline + high-dose meropenem (the 'meropenem sequence'). Two treatment sequences were analysed at a time; the CAZ-AVI sequence vs. the ceftolozane/tazobactam sequence; and the CAZ-AVI sequence vs. the meropenem sequence.

\section{Model inputs and data sources}

Model inputs were obtained primarily from clinical studies, published literature, and publicly available databases. Table 1 summarizes model inputs and data sources pertaining to baseline pathogens and their resistance rate for each treatment sequence. The analysis was based on the five most frequent baseline pathogens observed in the RECLAIM study. Inputs on resistance rates of CAZAVI plus metronidazole and meropenem were calculated in a forecast model obtained from 2017 resistance data for Italy $[17,20]$ and were validated by clinical experts.

Other model inputs and data sources are given in Table 2. Treatment efficacy was assessed by clinical evaluation at different stages, via response achieved at EOT visit, clinical cure achieved at TOC (i.e., first follow-up) visit, and recurrence of the infection observed at LFU (i.e., second follow-up) visit. Only SAEs which had relevant cost impact and may have resulted in treatment discontinuation or treatment switch were considered. Treatment efficacy and safety inputs for CAZ-AVI plus metronidazole and meropenem were obtained from the RECLAIM clinical trial, while those for ceftolozane/ tazobactam plus metronidazole were sourced from published literature [35]; those same inputs for the secondline treatment of colistin + tigecycline + high-dose meropenem were based on expert opinion. Classification of in-hospital mortality of a patient was made depending on appropriateness of the empirical treatment and resistance to it. In-hospital death rates were sourced from published literature, as there were only small death counts observed in the clinical trials. Treatment duration inputs were based on product labels.

The daily costs of CAZ-AVI, colistin + tigecycline + high-dose meropenem, and ceftolozane/tazobactam plus metronidazole were available on the official site of the Agenzia Italiana del Farmaco (AIFA), the Italian medicine agency [31]. However, the cost of colistin was not available in the AIFA database, so was taken from British National Formulary [36]. Hospitalisation costs in the model were calculated based on hospital length of stay and the proportion of time spent in intensive care unit vs. general ward. These inputs were categorized based on whether the patient achieved clinical cure. Costs of treatment-related SAEs were estimated as a one-time cost based on weighted average cost of various types of SAEs as observed in the RECLAIM study.

\section{Analyses}

\section{Base-case analysis}

In the base-case analysis, a 5-year time horizon was considered to cover the episode of the infection and the long-term impact. A 3\% annual discount rate was applied in the model to both costs and health benefits [10]. Two pairwise comparisons were performed to compare 1) the 
Table 1 Baseline pathogens and resistance rate for each treatment sequence

\begin{tabular}{|c|c|c|c|c|}
\hline \multirow[t]{2}{*}{ Pathogens } & \multirow{2}{*}{$\begin{array}{l}\text { Frequency } \\
\text { of baseline } \\
\text { pathogens }\end{array}$} & \multicolumn{3}{|l|}{ Resistance rate by pathogens } \\
\hline & & $\overline{C A Z-A V I}+$ metronidazole $^{b}$ & Ceftolozane/Tazobactam + metronidazole ${ }^{b}$ & Meropenem $^{b}$ \\
\hline Escherichia coli & $81 \%$ & $1 \%$ & $0 \%^{c}$ & $1 \%$ \\
\hline Streptococcus anginosus group & $15 \%$ & $0 \%^{c}$ & $0 \%^{c}$ & $0 \%^{c}$ \\
\hline Klebsiella pneumoniae & $13 \%$ & $1 \%$ & $52 \%^{d}$ & $52 \%$ \\
\hline Bacteroides fragilis & $12 \%$ & $0 \%^{c}$ & $0 \%^{c}$ & $0 \%^{c}$ \\
\hline Pseudomonas aeruginosa & $11 \%$ & $7 \%$ & $7 \%{ }^{e}$ & $24 \%$ \\
\hline
\end{tabular}

CAZ-AVI = ceftazidime-avibactam.

${ }^{a}$ Five most frequently identified baseline pathogens in the RECLAIM study

b2017 resistance data for Italy, calculated from forecast model [17, 20], and expert opinion

${ }^{c}$ Assumption, due to lack of evidence

${ }^{\mathrm{d} G i v e n}$ that ceftolozane/tazobactam has no coverage for $K$. pneumoniae carbapenemase (KPC), the base case was assumed using the resistance rate of $K$. pneumoniae to carbapenems

expert opinion

Table 2 Model inputs and data sources

\begin{tabular}{|c|c|c|c|c|}
\hline & CAZ-AVI + metronidazole & Ceftolozane/Tazobactam + metronidazole & Meropenem & $\begin{array}{l}\text { Colistin }+ \text { tigecycline }+ \text { high-dose } \\
\text { Meropenem }\end{array}$ \\
\hline $\begin{array}{l}\text { Probability of clinical } \\
\text { cure }^{a}\end{array}$ & $91.7 \%^{\mathrm{b}}$ & $94.1 \%^{\mathrm{c}}$ & $92.5 \%{ }^{\mathrm{b}}$ & $75.0 \%^{d}$ \\
\hline Probability of $A E^{e}$ & $4.9 \%^{\mathrm{e}}$ & $8.1 \%^{\mathrm{c}}$ & $3.8 \%^{\mathrm{e}}$ & $14.8 \%^{f}$ \\
\hline Probability of recurrence & $0.0 \%^{b}$ & $0.0 \%^{9}$ & $0.6 \%^{b}$ & $0.0 \%^{9}$ \\
\hline Treatment duration & 9.5 days $^{\mathrm{h}}$ & 9.0 days $^{\mathrm{h}}$ & 9.5 days $^{\mathrm{h}}$ & 9.5 days $^{\text {h }}$ \\
\hline \multicolumn{5}{|c|}{ Probability of in-hospital death ${ }^{i}$} \\
\hline \multicolumn{5}{|c|}{$\begin{array}{l}\text { Appropriate empiric treatment: } 4.80 \% \\
\text { Inappropriate empiric therapy: } 10.70 \% \\
\text { Resistant to empiric therapy: } 12.84 \%{ }^{j}\end{array}$} \\
\hline \multicolumn{5}{|l|}{ Utility (quality of life) } \\
\hline \multicolumn{5}{|c|}{$\begin{array}{l}\text { With clinical response: } 0.92^{k} \\
\text { Without clinical response: } 0.61^{1}\end{array}$} \\
\hline \multicolumn{5}{|l|}{ Hospital length of stay ${ }^{m}$} \\
\hline \multicolumn{5}{|c|}{$\begin{array}{l}\text { With clinical response: } 11.71 \text { days } \\
\text { Without clinical response: } 24.13 \text { days }\end{array}$} \\
\hline \multicolumn{5}{|c|}{ Proportion of hospitalisation days in ICU b } \\
\hline \multicolumn{5}{|c|}{$\begin{array}{l}\text { With clinical cure: } 26.92 \% \\
\text { With clinical failure: } 11.45 \%\end{array}$} \\
\hline $\begin{array}{l}\text { Daily drug costs, } \\
\text { (average daily dose) }\end{array}$ & $\begin{array}{l}€ 300.77 \text { (CAZ-AVI } 7500 \text { mg; } \\
\text { metronidazole } 1500 \mathrm{mg} \text { ) }\end{array}$ & $\begin{array}{l}€ 248.97 \text { (ceftolozane/tazobactam } 1500 \text { mg; } \\
\text { metronidazole } 1500 \text { mg) }\end{array}$ & $\begin{array}{l}€ 55.32 \\
(3000 \mathrm{mg})\end{array}$ & $\begin{array}{l}€ 218.55 \text { (colistin [IV] } 5 \text { mg; tigecycline } 100 \\
\text { mg; meropenem } 6000 \text { mg) }\end{array}$ \\
\hline Hospital cost per day & & General ward: $€ 697.23^{\circ} ; \mathrm{ICU} € 1383.00^{\mathrm{p}}$ & & \\
\hline Cost of $\mathrm{SAE}^{\circ}$ & & $€ 3027$ & & \\
\hline Cost of recurrence $^{\circ}$ & & $€ 6787$ & & \\
\hline
\end{tabular}

$\mathrm{AE}=$ adverse event; $\mathrm{BNF}=$ British National Formulary; $\mathrm{CAZ}-\mathrm{AVI}=$ ceftazidime-avibactam; ICU = intensive care unit; IV = intravenous; $\mathrm{SAE}=$ serious adverse event. ${ }^{\text {aP }}$ robability of clinical cure of patients without resistance

${ }^{\mathrm{b}} \mathrm{RECLAIM}$ clinical study data [1]

'Solomkin et al. 2015 [35]

dExpert opinion

${ }^{\mathrm{e}} \mathrm{AEs}$ considered in the model included only serious AEs, as these have relevant cost impact and can result in treatment discontinuation or treatment switch. Probability of SAE (up to EOT) was based on RECLAIM clinical study data

fPooled data from multiple sources: Chen et al. 2010 [21], Fomin et al. 2005 [22], Oliva et al. 2005 [23], Qvist et al. 2012 [24], and Towfigh et al. [25]

${ }^{\mathrm{g}}$ Assumption (due to lack of data)

${ }^{h}$ European Medicines Agency (EMA) product labels [26, 27]

iSturkenboom et al. 2005 [28]

${ }^{\mathrm{j}}$ Assumed to be $20 \%$ higher than mortality among patients with inappropriate empiric therapy (without resistance)

kSong et al. 2012 [29]

'Delate et al. 2001 [30], assuming similar utility for patients with different infections. Deterministic sensitivity analysis showed small impact of utility of clAl (i.e., utility applied while patients have not been cured) on the results

mpayer Analysis data

"nAIFA, Agenzia Italiana del Farmaco. 2014 (except for cost of colistin which was taken from BNF, converted to Euros using an exchange rate of $£ 1=€ 1.36$ ) [31]

oItalian hospital diagnosis-related groups (DRGs 2013 and 2015) [32, 33]

${ }^{\mathrm{P}}$ Tan et al. 2012 [34] 
CAZ-AVI sequence vs. ceftolozane/tazobactam sequence and 2) the CAZ-AVI sequence vs. meropenem sequence.

\section{Probabilistic sensitivity analysis}

The robustness of outcome results with regards to model uncertainty was studied through probabilistic sensitivity analysis (PSA) by using second-order Monte Carlo simulation and running the model for 100 simulations. A probability distribution was assigned to each parameter (i.e., costs and outcomes) to generate the inputs and to calculate the cost and effectiveness outcomes of each treatment sequence. Costs relating to use of healthcare resources were assumed to follow gamma distributions, while inputs limited to between zero and one (like probabilities and utilities) were assumed to follow a beta distribution. The standard error for some of these parameters was assumed to equal $10 \%$ of the mean because of lack of information on their variability. Cost-effectiveness acceptability curves were obtained by plotting the data of the probabilistic analysis on the cost-effectiveness plane.

\section{One-way deterministic sensitivity analyses}

Key model parameters were identified using one-way deterministic sensitivity analyses (DSA), where each parameter was varied by $\pm 20 \%$ of the base-case values while holding all other parameters constant. The results were defined in terms of incremental net benefit (INB), calculated as the difference of the incremental QALYs multiplied by the willingness-to-pay (WTP) threshold and the incremental costs, and were presented in the form of tornado diagrams.

\section{Scenario analyses}

Two scenario analyses were performed to test the assumptions used in the model. In one scenario (the 'no resistance adjustments' scenario), we removed the additional economic burden we had assumed in the base case to account for resistance to empiric antibiotics, such as an increase of in-hospital death rate, an increase in daily hospitalisation cost, and a decrease in efficacy of the second-line treatment. In the other (the ' $100 \%$ cure in second-line' scenario), efficacy of the second-line treatment was set to $100 \%$ (i.e., assuming patients were switched to the 'right' treatment once the resistant pathogens were identified from microbiological results).

\section{Results}

Comparison: CAZ-AVI sequence vs. Ceftolozane/

Tazobactam sequence

Base-case results

Results of the key base-case analysis comparing the CAZ-AVI sequence with the ceftolozane/tazobactam sequence are presented in Table 3 and Fig. 2. The proportion of patients cured was comparable between CAZ-AVI sequence and ceftolozane/tazobactam sequence
(93.04\% vs. 91.52\%, respectively). Consequently, a slightly lower proportion of in-hospital deaths due to infection was observed with the CAZ-AVI sequence, thus resulting in a slight increase in LYs and QALYs (0.027 LY and 0.039 QALY per patient) over the 5-year time horizon. A higher number of cures with the CAZ-AVI sequence led to a reduction in the average length of hospital stay $(0.38$ days per patient) (Table 3).

Results of cost outcomes suggested that there was an incremental cost of $€ 161$ per patient with the CAZ-AVI sequence. Higher drug costs (estimated increase of $€ 628$ per patient) were offset by a reduction in hospitalisation costs (estimated decrease of $€ 426$ per patient). The costs of treatment-associated SAEs were also lower in the CAZ-AVI sequence (average decrease of €41) (Fig. 2). Overall, the ICER was estimated at $€ 4099$ per QALY gained, which was well below the WTP threshold of $€ 30$, 000 per QALY in Italy (Table 3).

\section{Scenario analyses results}

In the 'no resistance adjustments' scenario, the ICER increased to $€ 11,461$ per QALY. However, the CAZ-AVI sequence is still considered a cost-effective option given the WTP threshold of $€ 30,000$ per QALY accepted in Italy. Results for the ' $100 \%$ cure in second-line' scenario suggested that the assumption of $100 \%$ efficacy for the second-line treatment had no impact on the ICER, as

Table 3 Clinical and economic outcomes for CAZ-AVI sequence vs. Ceftolozane/Tazobactam sequence (discounted by 3\%)

\begin{tabular}{|c|c|c|}
\hline Outcomes & CAZ-AVI sequence ${ }^{a}$ & $\begin{array}{l}\text { Ceftolozane/Tazobactam } \\
\text { sequence }^{\text {b }}\end{array}$ \\
\hline$\%$ of patients with cure & $93.04 \%$ & $91.52 \%$ \\
\hline $\begin{array}{l}\% \text { of patients died in } \\
\text { hospital }\end{array}$ & $5.02 \%$ & $5.60 \%$ \\
\hline$\%$ of patients with $\mathrm{AE}$ & $6.12 \%$ & $7.48 \%$ \\
\hline $\begin{array}{l}\text { Average number of days } \\
\text { in hospital }\end{array}$ & 13.04 & 13.42 \\
\hline LYS & 4.411 & 4.384 \\
\hline QALYS & 4.021 & 3.982 \\
\hline Drug costs & $€ 2952$ & $€ 2324$ \\
\hline Hospitalisation costs & $€ 11,355$ & $€ 11,781$ \\
\hline SAE costs & $€ 185$ & $€ 226$ \\
\hline Recurrence costs & $€ 0$ & $€ 0$ \\
\hline Total costs & $€ 14,492$ & $€ 14,331$ \\
\hline $\begin{array}{l}\text { Incremental cost per } \\
\text { QALY gained }\end{array}$ & & $€ 4099$ \\
\hline
\end{tabular}

$\mathrm{AE}=$ adverse event; $\mathrm{CAZ}-\mathrm{AVI}=$ ceftazidime-avibactam; $\mathrm{LY}=$ life year; $\mathrm{QALY}=$ quality-adjusted life year; $S A E=$ serious adverse event.

${ }^{a}$ CAZ-AVI plus metronidazole, followed by colistin + tigecycline + high-dose meropenem

${ }^{\mathrm{b}}$ Ceftolozane/tazobactam plus metronidazole, followed by colistin + tigecycline + high-dose meropenem 


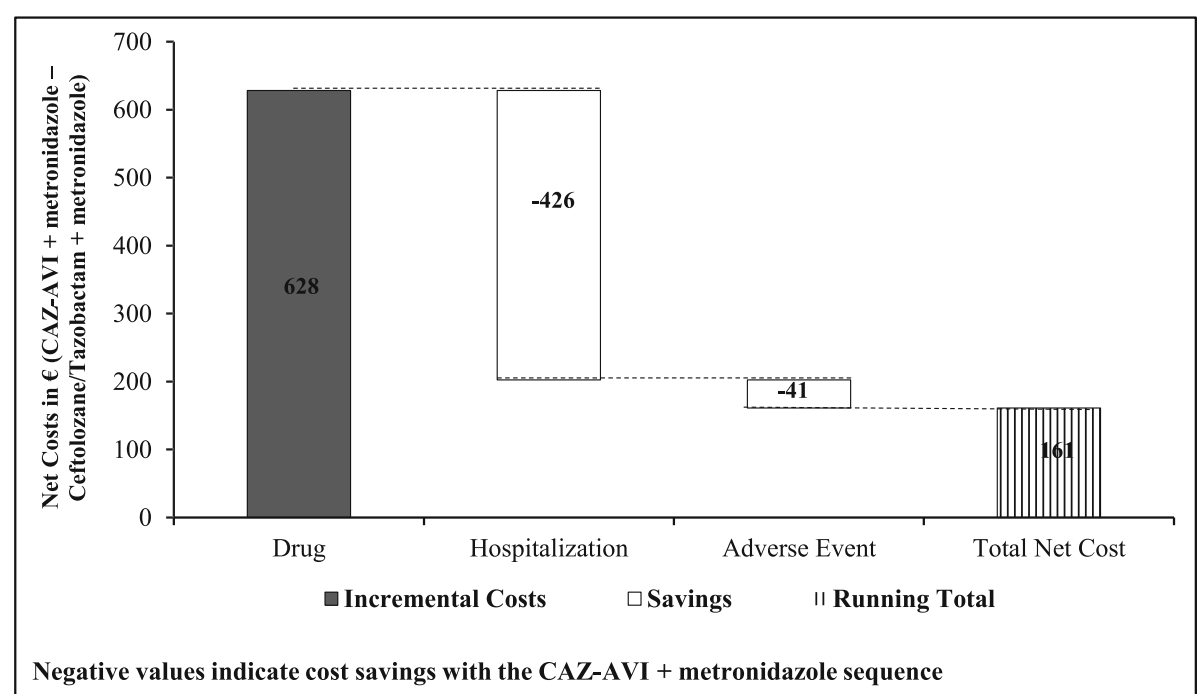

Fig. 2 Incremental cost outcomes per patient for CAZ-AVI sequence vs. Ceftolozane/Tazobactam sequence. Abbreviations: CAZ-AVI ceftazidime-avibactam. CAZ-AVI sequence: CAZ-AVI plus metronidazole, followed by colistin + tigecycline + high-dose meropenem. Ceftolozane/tazobactam sequence: Ceftolozane/tazobactam plus metronidazole, followed by colistin + tigecycline + high-dose meropenem

the ICER changed by less than $1 \%$ compared to the base case (Table 4).

\section{Deterministic sensitivity analysis results}

Results of the DSA comparing the CAZ-AVI sequence with the ceftolozane/tazobactam sequence are presented as a tornado diagram (Fig. 3). The 10 parameters that most influenced the INB (calculated based on a WTP threshold of $€ 30,000$ per QALY) are included and presented in their order of the influence. In the base case, the CAZ-AVI sequence was estimated with an INB of $€ 1019$, suggesting that the CAZ-AVI sequence was cost effective (i.e., positive INB indicates cost effectiveness) in comparison to the ceftolozane/tazobactam sequence. Results from the DSA showed that variation in response rates at the EOT

Table 4 Scenario analyses results for CAZ-AVI sequence vs. Ceftolozane/Tazobactam sequence

\begin{tabular}{ll}
\hline Scenario & $\begin{array}{l}\text { Incremental cost per QALY } \\
\text { gained (\% change from base case) }\end{array}$ \\
\hline Base case & $€ 4099$ \\
Resistance adjustments & \\
No adjustments & $€ 11,461(+180 \%)$ \\
$\begin{array}{l}\text { Second-line efficacy } \\
\text { Assumed 100\% response/ } \\
\text { cure rates in treatment }\end{array}$ & $€ 4060(-1 \%)$ \\
\hline
\end{tabular}

CAZ-AVI = ceftazidime-avibactam; $Q A L Y=$ quality-adjusted life year. CAZ-AVI sequence: CAZ-AVI plus metronidazole, followed by colistin + tigecycline + high-dose meropenem. Ceftolozane/tazobactam sequence: Ceftolozane/tazobactam, followed by colistin + tigecycline + high-dose meropenem assessment and the clinical cure rates at the TOC visit highly influenced the outcomes. The INB increased when response and cure rates for the CAZ-AVI sequence were increasing or decreasing those for the ceftolozane/tazobactam sequence. Other parameters had a moderate influence on the INB. The INB results became negative, (i.e., the CAZ-AVI sequence was not cost effective at the threshold of $€ 30,000$ per QALY), when the cure and response rates of CAZ-AVI were decreased and when the response rate of ceftolozane/tazobactam increased by $10 \%$.

\section{Probabilistic sensitivity analysis results}

The PSA results showed that in $45 \%$ of the runs, the CAZAVI sequence was dominant, producing higher numbers of QALYs at lower costs compared to the ceftolozane/tazobactam sequence, as illustrated in Fig. 4 by the clustering of the majority of the iterations in the South-East quadrant. In $26 \%$ of the iterations, the CAZ-AVI sequence was found to be costlier but still produced a higher number of QALYs, with a second cluster in the North-East quadrant of Fig. 4.

The cost-effectiveness acceptability curves (CEAC) appearing in Fig. 5 suggests that the CAZ-AVI sequence had a higher percentage of being cost effective (ranging from 55 to $65 \%$ ) compared to the ceftolozane/tazobactam sequence for all WTP thresholds explored (up to $€ 100,000$ per QALY).

\section{Comparison: CAZ-AVI sequence vs. Meropenem sequence Base-case results}

The model suggested better health outcomes for the CAZ-AVI sequence, with $92.98 \%$ of patients in the 


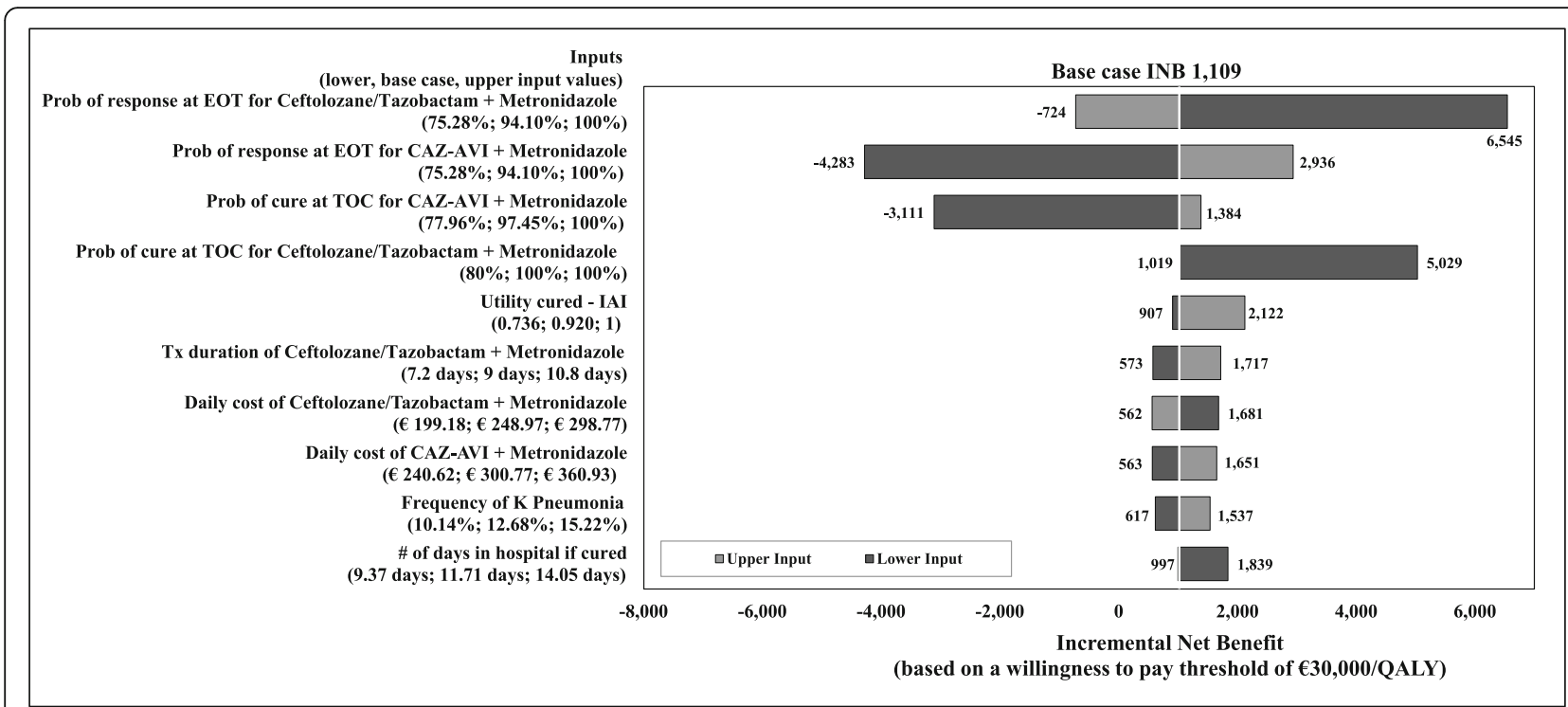

Fig. 3 One-way deterministic sensitivity analysis for CAZ-AVI sequence vs. Ceftolozane/Tazobactam sequence, INB based on a willingness-to-pay threshold. Abbreviations: CAZ-AVI ceftazidime-avibactam, CIAI complicated intra-abdominal infection, EOT end-of-treatment, INB incremental net benefit, Prob probability, TOC test-of-cure, Tx treatment. CAZ-AVI sequence: CAZ-AVI plus metronidazole, followed by colistin + tigecycline + high-dose meropenem. Ceftolozane/tazobactam sequence: Ceftolozane/tazobactam plus metronidazole, followed by colistin + tigecycline + high-dose meropenem. Note: A positive INB indicates the CAZ-AVI sequence is cost effective compared to the ceftolozane/ tazobactam sequence, and vice versa

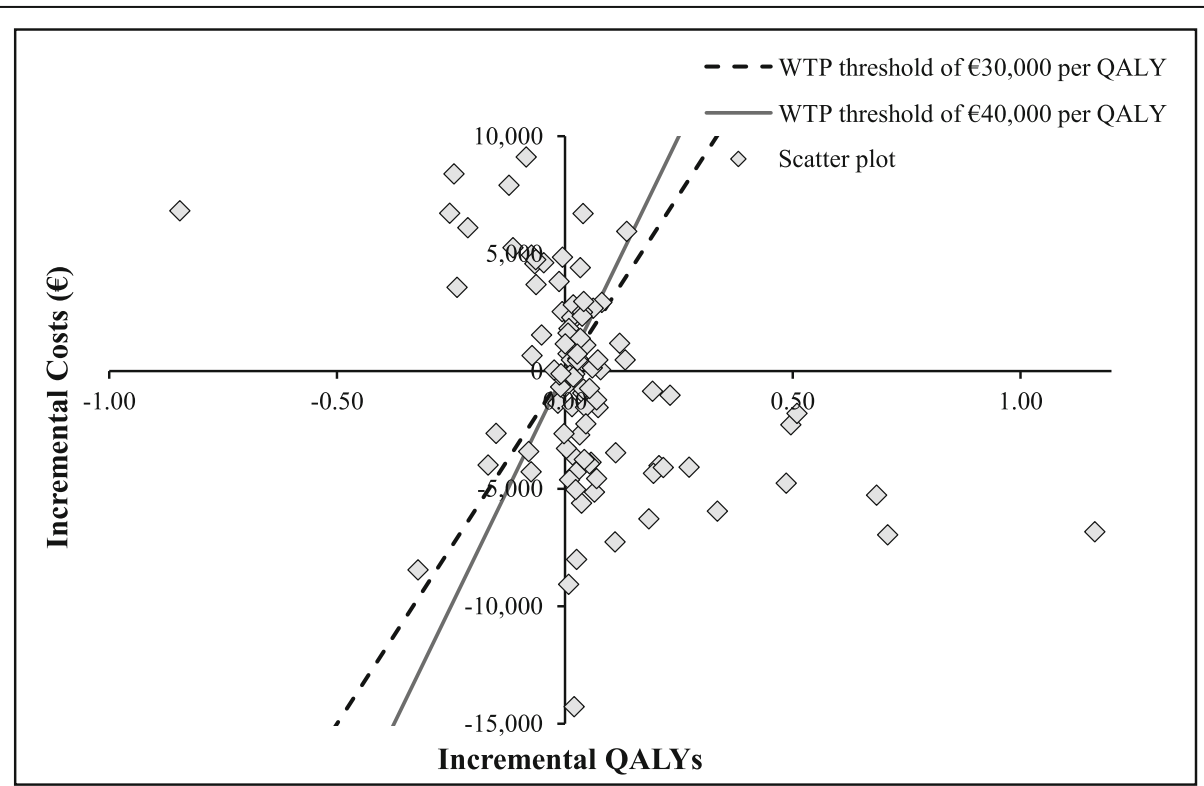

Fig. 4 Results from probabilistic sensitivity analysis for CAZ-AVI sequence vs. Ceftolozane/Tazobactam sequence, on cost-effectiveness plane. Abbreviations: CAZ-AVI, ceftazidime-avibactam; QALY, quality-adjusted life year; WTP, willingness-to-pay. CAZ-AVI sequence: CAZ-AVI plus metronidazole, followed by colistin + tigecycline + high-dose meropenem. Ceftolozane/tazobactam sequence: Ceftolozane/tazobactam plus metronidazole, followed by colistin + tigecycline + high-dose meropenem. Notes: Each dot represents the cost-effectiveness outcome from each iteration. The threshold lines represent cost-effectiveness thresholds of $€ 30,000$ or $€ 40,000$ per QALY (i.e., the maximum amount society is willing to pay for a QALY gained). In cases that fall to the right and below this line, the CAZ-AVI sequence is cost effective compared to the ceftolozane/ tazobactam sequence. In cases that fall to left and above this line, the CAZ-AVI sequence is not cost effective compared to the ceftolozane/tazobactam sequence 


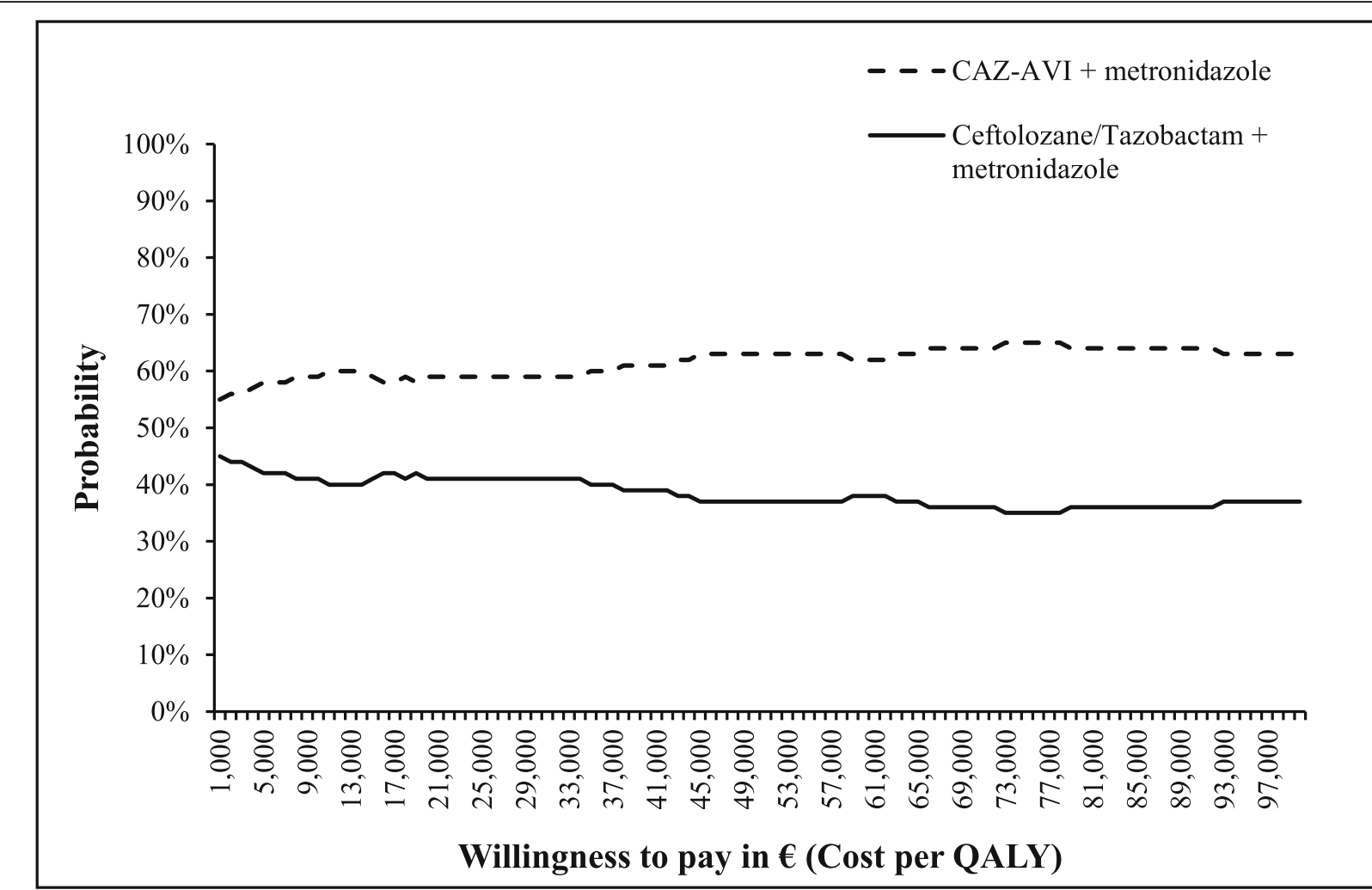

Fig. 5 Results from probabilistic sensitivity analysis for CAZ-AVI sequence vs. Ceftolozane/Tazobactam sequence, on cost-effectiveness acceptability curve. Abbreviations: CAZ-AVI ceftazidime-avibactam, QALY quality-adjusted life year. CAZ-AVI sequence: CAZ-AVI plus metronidazole, followed by colistin + tigecycline + high-dose meropenem. Ceftolozane/tazobactam sequence: Ceftolozane/tazobactam plus metronidazole, followed by colistin + tigecycline + high-dose meropenem

CAZ-AVI sequence achieving clinical cure compared to $90.24 \%$ of patients receiving the meropenem sequence. Time spent in hospital per patient was also lower in the CAZ-AVI sequence $(\Delta=-1.24$ days per patient $)$ (Table 5). Patients treated with the CAZ-AVI sequence had higher number of QALYs as well $(\Delta=0.059$ QALYs per patient).

The average total drug costs with the CAZ-AVI sequence were higher by $€ 2125$ per patient, mainly due to the higher drug acquisition cost of CAZ-AVI. However, the better clinical outcomes of the CAZAVI sequence meant that patients stayed in hospital an average of 1.24 days less than those receiving the meropenem sequence, reducing hospitalisation costs by $€ 1191$ per patient. Overall, the net incremental cost was $€ 924$ per patient in the CAZ-AVI sequence (Fig. 6). The ICER for the CAZ-AVI sequence was estimated at $€ 15,574$ per QALY gained, again well below the $€ 30,000$ per QALY WTP threshold accepted in Italy.

\section{Scenario results}

In the conservative scenario, when we removed the additional impact of resistance on hospitalisation
Table 5 Clinical and economic outcomes for CAZ-AVI sequence vs. Meropenem sequence, discounted by 3\%

\begin{tabular}{lcc}
\hline Outcomes & $\begin{array}{c}\text { CAZ-AVI } \\
\text { Sequence }^{\text {a }}\end{array}$ & $\begin{array}{c}\text { Meropenem } \\
\text { Sequence }^{\text {b }}\end{array}$ \\
\hline \% of patients with cure & $92.98 \%$ & $90.24 \%$ \\
\% of patients died in hospital & $5.04 \%$ & $5.68 \%$ \\
\% of patients with AE & $6.14 \%$ & $5.74 \%$ \\
Average days in hospital & 12.94 & 14.18 \\
LYs & 4.410 & 4.381 \\
QALYs & 4.019 & 3.960 \\
Drug costs & $€ 2943$ & $€ 818$ \\
Hospitalisation costs & $€ 11,262$ & $€ 12,453$ \\
SAE costs & $€ 186$ & $€ 174$ \\
Recurrence costs & $€ 0$ & $€ 22$ \\
Total costs & $€ 14,391$ & $€ 13,467$ \\
Incremental cost per & & $€ 15,574$ \\
QALY gained & & \\
\hline
\end{tabular}

$\mathrm{AE}=$ adverse event; $\mathrm{CAZ}-\mathrm{AVI}=$ ceftazidime-avibactam; $\mathrm{LY}=$ life year; $\mathrm{QALY}=$ quality-adjusted life year; $\mathrm{SAE}=$ serious adverse event.

${ }^{a}$ CAZ-AVI plus metronidazole, followed by colistin + tigecycline + high-dose meropenem

${ }^{\mathrm{b}}$ Meropenem, followed by colistin + tigecycline + high-dose meropenem 


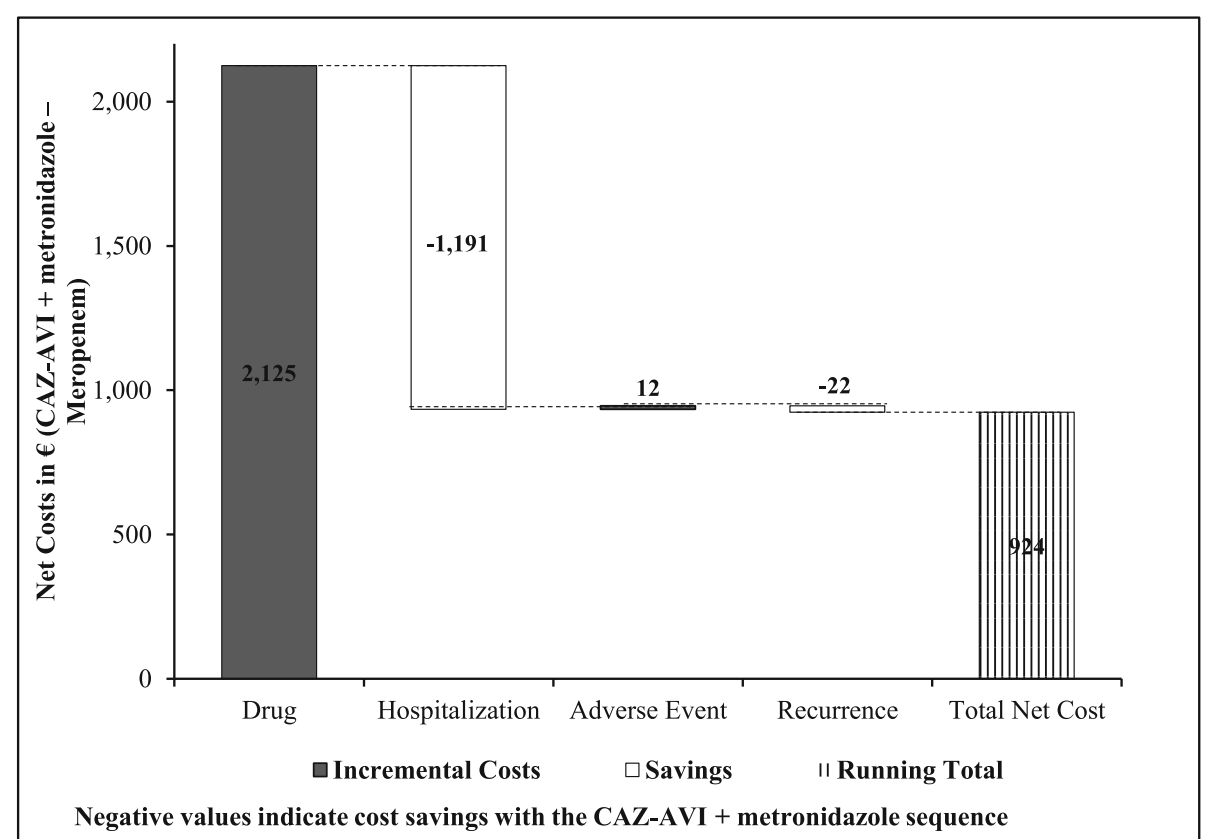

Fig. 6 Incremental cost outcomes per patient for CAZ-AVI sequence vs. meropenem sequence. Abbreviations: CAZ-AVI ceftazidime-avibactam, CAZ-AVI sequence: CAZ-AVI plus metronidazole, followed by colistin + tigecycline + high-dose meropenem. Meropenem sequence: Meropenem, followed by colistin + tigecycline + high-dose meropenem

costs, mortality, and cure rates of the subsequent treatment, the ICER was observed to increase to $€ 27$, 626 per QALY, still below Italy's $€ 30,000$ per QALY threshold. However, in the ' $100 \%$ cure in second-line' scenario (in which the response/cure rates of secondline treatment were assumed to be $100 \%$ ), the ICER increased to $€ 30,614$ per QALY, slightly above the threshold (Table 6).

\section{Deterministic sensitivity analysis results}

Results of the DSA comparing the CAZ-AVI with the meropenem sequence are presented as a tornado diagram (Fig. 7), showing the 10 parameters that most

Table 6 Scenario analyses results for CAZ-AVI sequence vs. Meropenem sequence

\begin{tabular}{ll}
\hline Scenario & $\begin{array}{l}\text { Incremental cost per QALY gained } \\
\text { (\% change from base case) }\end{array}$ \\
\hline Base case & $€ 15,574$ \\
Resistance adjustments & \\
$\quad$ No adjustments & $€ 27,626(+77 \%)$ \\
$\begin{array}{l}\text { Second-line efficacy } \\
\text { Assumed 100\% response/ } \\
\text { cure rates in treatment }\end{array}$ & $€ 30,614(+97 \%)$ \\
\hline
\end{tabular}

CAZ-AVI = ceftazidime-avibactam; $Q A L Y=$ quality-adjusted life year. CAZ-AVI sequence: CAZ-AVI plus metronidazole, followed by colistin + tigecycline + high-dose meropenem. Ceftolozane/tazobactam sequence: Ceftolozane/tazobactam plus metronidazole, followed by colistin + tigecycline + high-dose meropenem influenced the INB (based on a WTP threshold of $€ 30$, 000 per QALY) presented in their order of influence. In the base case, the CAZ-AVI sequence was cost effective (with a positive INB of $€$ 856) in comparison to the meropenem sequence. Results from the DSA showed that varying the response rates at the EOT assessment and the clinical cure rates at the TOC visit influenced the outcomes most. The INB was observed to increase when response and cure rates for CAZ-AVI sequence were increased or when those for the meropenem sequence were decreased. Also, among the 10 most influential inputs are the drug cost of CAZ-AVI plus metronidazole, the number of hospitalisation days with treatment failure, frequencies of $K$. pneumoniae and of E. coli, and utilities. The CAZ-AVI sequence was not cost effective (i.e., INB was negative) when we lowered utility score for 'cure' (from 0.92 in the base case to 0.74), the rate of response at EOT or of clinical cure at TOC for the CAZAVI sequence, and when we increased response rate at EOT for the meropenem sequence.

\section{Probability sensitivity analysis results}

The PSA results showed that in $41 \%$ of the runs, the CAZ-AVI sequence was more effective, producing a higher number of QALYs with higher costs compared to the meropenem sequence, as illustrated in Fig. 8, where the majority of the iterations lie in the North-East quadrant. Additionally, the CAZ-AVI sequence was found to 


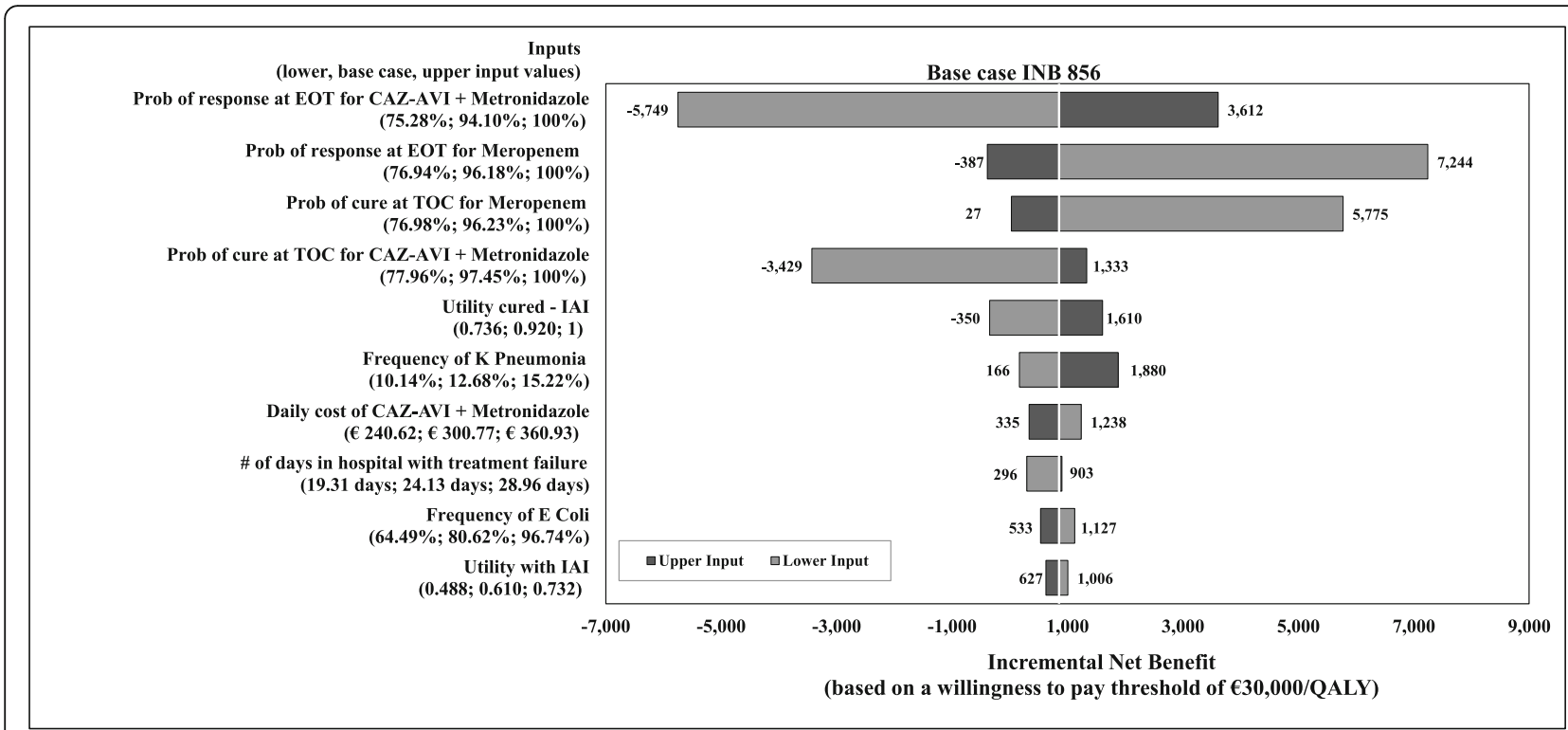

Fig. 7 One-way deterministic sensitivity analysis for CAZ-AVI sequence vs. meropenem sequence, INB based on a willingness-to-pay threshold, Abbreviations: CAZ-AVI ceftazidime-avibactam, CIAI complicated intra-abdominal infection, EOT end-of-treatment, INB incremental net benefit, Prob probability, TOC test-of-Cure. CAZ-AVI sequence: CAZ-AVI plus metronidazole, followed by colistin + tigecycline + high-dose meropenem. Meropenem sequence: Meropenem, followed by colistin + tigecycline + high-dose meropenem. Note: Positive INB indicates CAZ-AVI sequence was cost effective compared to meropenem sequence, and vice versa

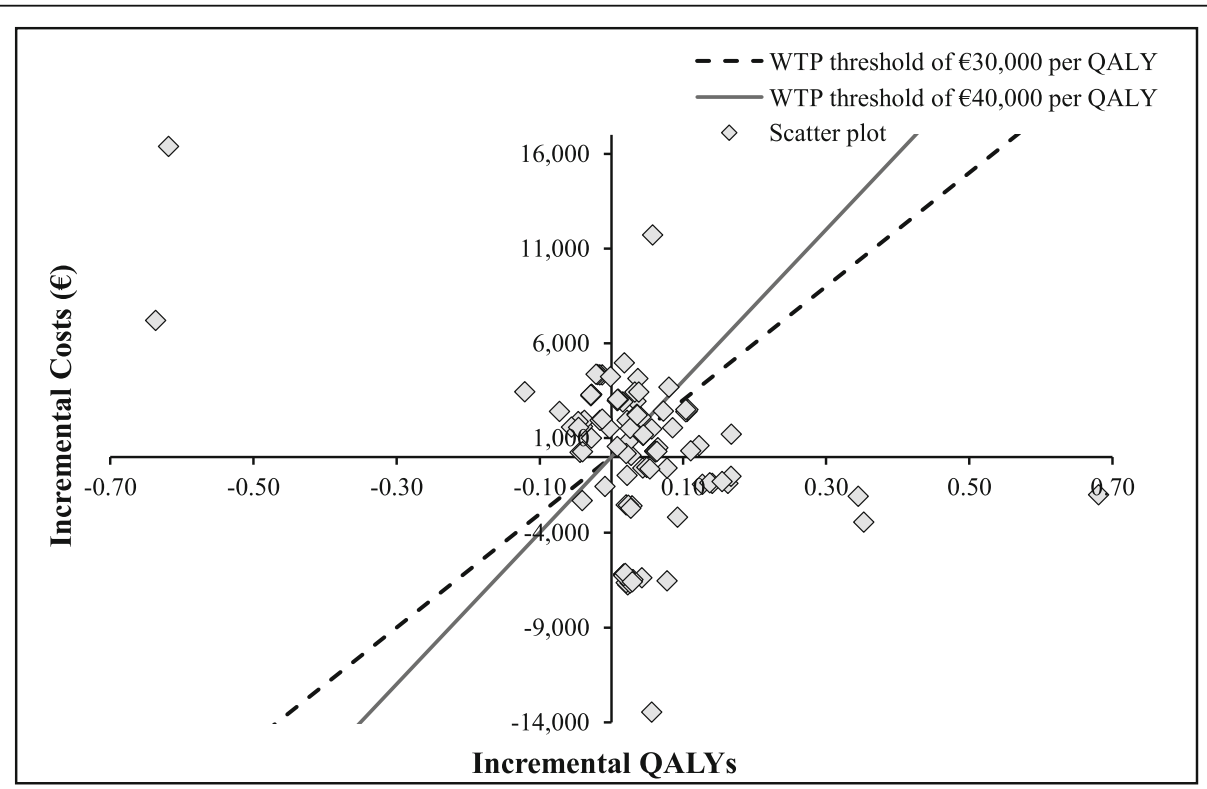

Fig. 8 Probabilistic sensitivity analysis for CAZ-AVI sequence vs. meropenem sequence (on cost-effectiveness plane). Abbreviations: CAZ-AVI ceftazidime-avibactam, QALY quality-adjusted life year, WTP willingness-to-pay. CAZ-AVI sequence: CAZ-AVI plus metronidazole, followed by colistin + tigecycline + high-dose meropenem. Meropenem sequence: Meropenem, followed by colistin + tigecycline + high-dose meropenem. Notes: Each dot represents cost-effectiveness outcome from each iteration. The threshold lines represent cost-effectiveness thresholds $(€ 30,000$ or $€ 40,000$ per QALY), the maximum amount society is willing to pay for a QALY gain. In cases that fall to the right and below this line, the CAZ$\mathrm{AVI}$ sequence is cost effective compared to the meropenem sequence. In cases that fall to left and above this line, the CAZ-AVI sequence is not cost effective compared to the meropenem sequence 


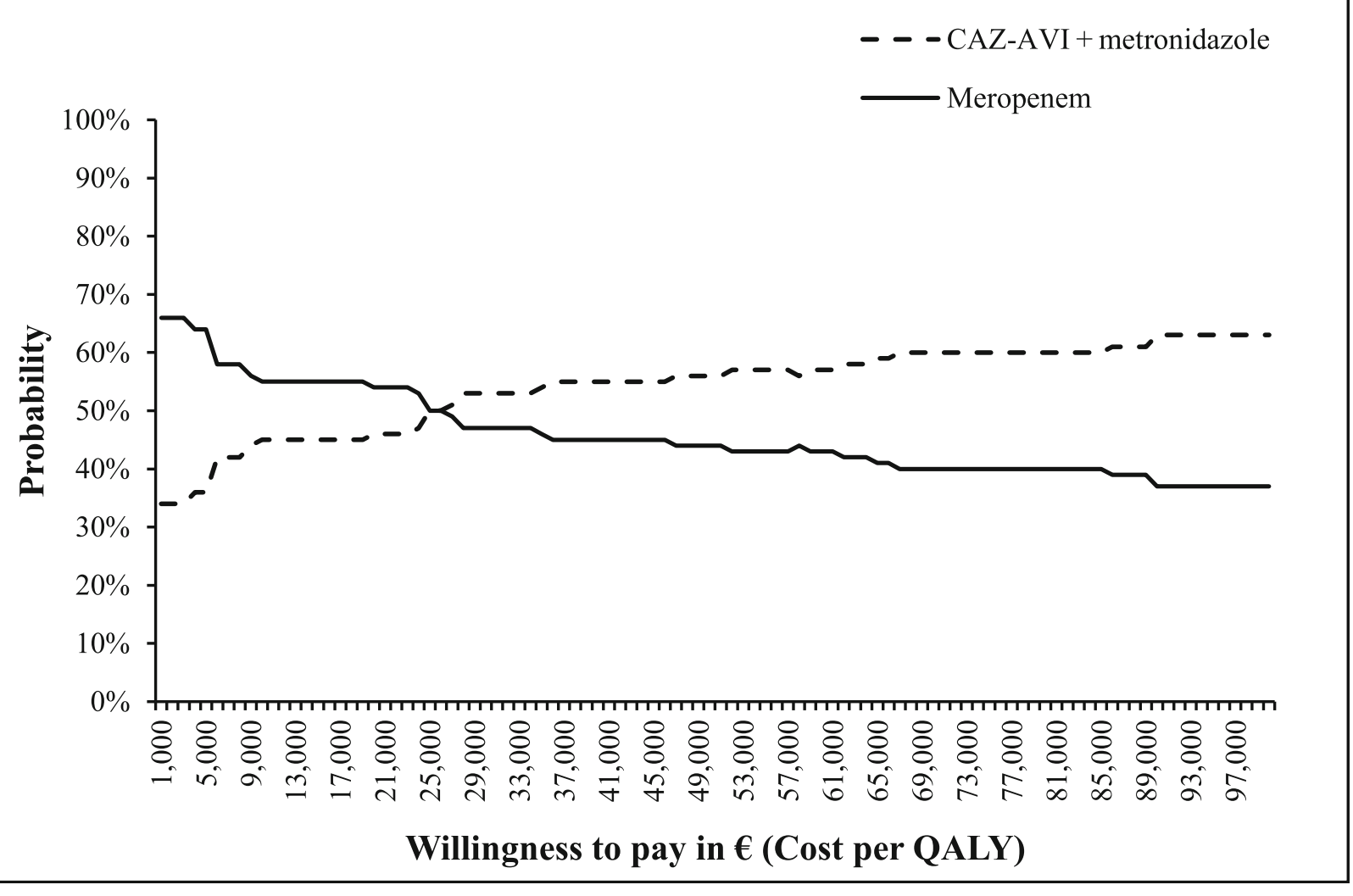

Fig. 9 Probabilistic sensitivity analysis for CAZ-AVI sequence vs. meropenem sequence (on cost-effectiveness acceptability curve). Abbreviations: CAZ-AVI ceftazidime-avibactam, QALY quality-adjusted life year. CAZ-AVI sequence: CAZ-AVI plus metronidazole, followed by colistin + tigecycline + high-dose meropenem. Meropenem sequence: Meropenem, followed by colistin + tigecycline + high-dose meropenem

be dominant in $32 \%$ of the iterations (higher QALYs with lower costs; runs appear in the South-East quadrant) and found to be dominated in $25 \%$ of the iterations (lower QALYs with higher costs; runs appear in the North-West quadrant).

The CEAC in Fig. 9 suggests that the CAZ-AVI sequence had a higher probability of being a cost-effective treatment sequence compared to the meropenem sequence when the WTP threshold was at least $€ 26,000$ per QALY.

\section{Discussion}

The impact of bacterial resistance on the cost effectiveness of antimicrobial therapy in cIAIs has not been systematically investigated, perhaps due to a lack of evidence. In this study, we analysed cost effectiveness of CAZ-AVI plus metronidazole as an empiric treatment in comparison to ceftolozane/tazobactam plus metronidazole and to meropenem for hospitalised patients with cIAI from the Italian publicly funded healthcare (thirdparty payer) perspective.

The base-case analysis suggested that empiric therapy with the CAZ-AVI sequence for patients hospitalised with cIAI was cost effective compared to both comparator sequences in terms of efficacy and cost outcomes. Clinical efficacy outcome data demonstrated that patients treated with the CAZ-AVI sequence had better clinical outcomes (i.e., higher proportion of patients cured, increased QALYs per patient, and reduced average length of hospital stays) than did those patients treated with either comparator sequence. The observed higher incremental costs related to drug acquisition and SAEs with the CAZ-AVI sequence were partly offset by the lower hospitalisation cost compared to the comparator sequences. The total cost outcomes for the CAZAVI sequence were comparatively higher $(€ 161$ and $€$ 924 incremental cost vs. the ceftolozane/tazobactam and the meropenem sequences, respectively). However, the ICERs for the CAZ-AVI sequence ( $€ 4099$ vs. the ceftolozane/tazobactam sequence and $€ 15,574$ vs. the meropenem sequence) were still well below the accepted WTP threshold in Italy of $€ 30,000$ per QALY. Scenario analyses show, when changing inputs in favour of the comparators - i.e., resistance adjustment factors are not considered, or second-line efficacy is assumed to be $100 \%$, the ICERs still remain below or exceed only by a 
bit the threshold of $€ 30,000$. Probabilistic sensitivity results demonstrate similar conclusions. When varying the input parameters of the model, the majority of the outcomes shows cost-effectiveness of CAZ-AVI sequence. Furthermore, in $45 \%$ (compared to ceftolozane/tazobactam sequence) and in $41 \%$ (compared to meropenem sequence) of the outcomes the CAZ-AVI sequence is dominant. Thus, the results indicate that CAZ-AVI can be a cost-effective alternative in treating patients with cIAI. Furthermore, a recent budget impact study demonstrated that including CAZ-AVI in the hospital formulary for treatment of cIAI in Italy had only a minimal impact $(0.74 \%$ increase over three years) on the total healthcare budget [37].

In Europe, the higher prevalence of multi-drug resistance in several pathogens, including the Enterococcus species, carbapenem-resistant $P$. aeruginosa and Acinetobacter baumannii, ESBL-producing E. coli, and Klebsiella species that are responsible for IAIs, has led to a corresponding increase in associated mortality and morbidity rates [38]. Hence, careful use of currently effective antimicrobial agents has become important to avoid and/or minimize emergence of resistance [38]. The results from our study indicate that using CAZ-AVI plus metronidazole as an empiric treatment among patients in the current resistance situation where resistance to CAZ-AVI has not yet developed resulted in patients receiving appropriate treatment earlier, and in better health outcomes overall. In addition, although not reflected in the economic outcomes of this study, the reduction in hospital stay provided by the CAZ-AVI sequence implicitly indicates additional bed days that hospitals can reallocate to other patients, and also a reduction in the risk of acquiring and/or transmitting new infections associated with prolonged hospital stay.

Our study is the first to look at the cost effectiveness of CAZ-AVI in cIAIs; however, several earlier models have examined cost effectiveness of antibiotics used in this indication. A patient-level simulation model published by Prabhu et al. in 2017 demonstrated that ceftolozane/tazobactam plus metronidazole was more cost effective and dominated piperacillin-tazobactam as an empiric treatment, due to lower total cost per patient (\$ 44,226 vs. \$ 44,811 respectively) and higher QALYs gained per patient (12.85 vs. 12.70), resulting in reduced length of hospital stay ( $\Delta=0.63$ days) per patient [5]. In an earlier study, Barie et al. compared the economic benefits of cefepime plus metronidazole with those of imipenem-cilastatin in the treatment of cIAIs; that study showed the cost effectiveness of cefepime [39].

Our model analysis has some important limitations to note. First, we had to predefine the treatment pathways because of the model structure; hence, the choices for subsequent treatment for each individual patient could not be defined. Second, the de-escalation of treatment of patients who are susceptible to the empiric treatment was not considered in the study. If step-down therapy had been included in the model, the results would have favoured CAZ-AVI given higher proportion of patients was susceptible to CAZ-AVI and thus treatment costs would have been lower. Therefore, this assumption can be considered conservative. Third, since the RECLAIM study and other published literature served as our clinical data source for efficacy and SAEs, other methods of data synthesis, such as indirect treatment comparisons or mixed treatment comparisons could not be used. Fourth, the available clinical data (efficacy and safety) were based on multi-centre clinical studies and thus were assumed to be applicable to Italy. Fifth, as AEs were captured as an aggregate, the $\mathrm{AE}$ unit cost we calculated was dependent on the distribution of the AEs observed in the CAZ-AVI clinical studies. Hence, a similar distribution of AEs was assumed for the other treatment sequences in the current model analysis. As shown in the results of this study, $\mathrm{AE}$ costs contributed only 1 to $2 \%$ to the total costs. Thus, the impact of this assumption on the results was considered minimal. Sixth, the model assumes all deaths occurring in the model are during hospital stay. Death after hospitalisation was not considered, as it was assumed that patients were cured a priori to hospital discharge. In addition, given the short time horizon death due to background mortality was not considered. These assumptions were applied to all treatment groups and thus were not expected to have significant impact on the results. Seventh, due to no available data on the utility of patients with cIAI at the time of the study, the model utilized published utility data of other infectious disease [30]. This input was tested in sensitivity analysis and was fond to have small impact on the results (Fig. 3 and Fig. 7). And lastly, given the antibiotic resistance rate can evolve over time, the results from our study may not be generalizable in the future.

Our study has several strengths to note as well. One, the model included a broad range of inputs, such as response, cure, treatment duration, hospitalisation, AEs, and infection recurrence, resulting in more realistic outcomes. Two, the impact of resistant pathogens, which cannot be demonstrated in the randomized clinical trials, was incorporated in the model, allowing us to demonstrate the real value of the antibiotic agents. The model allowed pathogen resistance to affect daily hospitalisation costs, mortality, and cure rates of second-line treatment. In a conservative scenario analysis that did not take the impact of resistance into account, the CAZAVI sequence still demonstrated its cost effectiveness despite the increase in the ICERs (i.e., the ICERs increased to $€ 11,461$ and $€ 27,626$ per QALY in the comparison of the CAZ-AVI sequence to the ceftolozane/ 
tazobactam and the meropenem sequences, respectively). Three, various efficacy aspects, including clinical response, cure, and recurrence were analysed in the model to delineate the required clinical and economical outcomes in detail. Four, the model uses a patient-level simulation, a well-established efficacy and economic model technique, whose 'treatment switch' allows the movement of patients from one treatment level to the next. This approach allows development of real-world models covering detailed treatment-related consequences (e.g., the impact of resistance). All these strengths allow the true economic value of CAZ-AVI to be captured in a way that the necessary non-inferiority design of antibiotic clinical trials cannot. The rationales for the non-inferiority design are not in question; the aim of clinical trials for antibiotics is not to identify superior treatments but to find those that are efficacious and safe for use as alternate treatments when existing agents are rendered ineffective by emerging antibiotic resistance.

Unlike drugs in other therapeutic areas such as oncology or cardiovascular disease, antibiotics do not seem to be economically valued at an appropriate level by society. CAZ-AVI and other novel antibiotics reduce mortality when used to treat life-threatening infections and therefore are lifesaving drugs [40].

\section{Conclusions}

In deciding on treatment for cIAIs, healthcare providers must consider a variety of treatment-related parameters, including local resistance data, efficacy, risk of AEs, and the resource burden associated with managing the infection. Choosing the appropriate empiric treatment is of high importance, since early and effective treatment not only results in better clinical outcomes, but also extends the lifetime of antibiotic agents in this period of rising pathogen resistance. The results from our study support that the combination of CAZ-AVI plus metronidazole is a suitable alternative to ceftolozane/tazobactam plus metronidazole and to meropenem, as it is expected to provide comparatively better clinical benefits (i.e., higher cure rates, shorter hospital stays, and improved quality of life) in a cost-effective manner for Italian patients with cIAI.

\footnotetext{
Abbreviations

CAZ-AVI: Ceftazidime/avibactam; clAls: Complicated intra-abdominal infections; EMA: European Medicines Agency; TOC: Test-of-cure; US: United States; EOT: End-of-treatment.; LFU: Long-term follow-up; AE: Adverse events; LYS: Life years; QALYs: Quality-adjusted life years; SAE: Serious adverse events; AIFA: Agenzia Italiana del Farmaco; PSA: Probabilistic sensitivity analysis; DSA: Deterministic sensitivity analyses; INB: Incremental net benefit; WTP: Willingness-to-pay; ICER: Incremental cost-effectiveness ratio; CEAC: Cost-effectiveness acceptability curves
}

\section{Authors' contributions}

TK and ET designed and implemented the model and analysed the results. RDV, NBP, and CC were involved in the design, data collection of the model and in the analysis of the results. CE and MB provided clinical experience on the model design and data inputs. All authors read and approved the final manuscript.

\section{Funding \\ This work was supported by Pfizer Inc. Pfizer also funded medical writing assistance by Evidera.}

\section{Availability of data and materials \\ The datasets used and/or analysed during the current study are available from the corresponding author on reasonable request.}

Ethics approval and consent to participate

Not Applicable.

Consent for publication

Not Applicable.

\section{Competing interests}

TK and ET are employees of Evidera, which received funding from Pfizer in connection with conducting the study, developing this manuscript, and medical writing. RDV, NBP, and CC are employees of Pfizer, and hold Pfizer stock and/or stock options. CE is an employee of Klinikum Peine, Academic Hospital of Medical University Hannover, (Hannover, Germany), and MB is an employee of University of Genoa, Genoa and Hospital Policlinico San Martino - IRCCS, Genoa (Italy), each of which received research funding from Pfizer. Outside the submitted work, MB has received funding for scientific advisory boards, travel and speaker honoraria from Angelini, AstraZeneca, Bayer, Cidara, Cepheid, Cubist, Pfizer, Menarini, MSD, Nabriva, Paratek, Roche, Shionogi, Tetraphase, The Medicine Company and Astellas Pharma Inc.

\section{Author details}

${ }^{1}$ Evidera, The Ark, 201 Talgarth Road, Hammersmith, London W6 8BJ, UK. ${ }^{2}$ Klinikum Peine, Academic Hospital of Medical University Hannover, Hannover, Germany. ${ }^{3}$ Infectious Diseases Clinic, Department of Health Sciences, University of Genoa, Genoa and Hospital Policlinico San Martino IRCCS, Genoa, Italy. ${ }^{4}$ Evidera, Bég u. 3-5 / 520, Budapest 1022, Hungary. ${ }^{5}$ Pfizer, Via Valbondione, 113, 00188 Rome, Italy. ${ }^{6}$ Pfizer, 23-25 Avenue du Dr Lannelongue, 75014 Paris, France.

Received: 6 September 2019 Accepted: 13 November 2019 Published online: 21 December 2019

\section{References}

1. Mazuski JE, Gasink LB, Armstrong J, Broadhurst H, Stone GG, Rank D, et al. Efficacy and safety of ceftazidime-avibactam plus metronidazole vs. meropenem in the treatment of complicated intra-abdominal infection: results from a randomized, controlled, double-blind, phase 3 program. Clin Infect Dis. 2016;62(11):1380-9. https://doi.org/10.1093/cid/ciw133.

2. Nicolau DP. Focus on ceftazidime-avibactam for optimizing outcomes in complicated intra-abdominal and urinary tract infections. Expert Opin Investig Drugs. 2015;24(9):1261-73. https://doi.org/10.1517/13543784. 2015.1062873.

3. Goodlet KJ, Nicolau DP, Nailor MD. Ceftolozane/tazobactam and ceftazidime/avibactam for the treatment of complicated intra-abdominal infections. Ther Clin Risk Manag. 2016;12:1811-26. https://doi.org/10.2147/ TCRM.S120811.

4. Lee YR, McMahan D, McCall C, Perry GK. Complicated intra-abdominal infections: the old antimicrobials and the new players. Drugs. 2015;75(18): 2097-117. https://doi.org/10.1007/s40265-015-0506-7.

5. Prabhu VS, Solomkin JS, Medic G, Foo J, Borse RH, Kauf T, et al. Costeffectiveness of ceftolozane/tazobactam plus metronidazole vs piperacillin/ tazobactam as initial empiric therapy for the treatment of complicated intra-abdominal infections based on pathogen distributions drawn from national surveillance data in the United States. Antimicrob Resist Infect Control. 2017;6:107. https://doi.org/10.1186/s13756-017-0264-2.

6. Zasowski EJ, Rybak JM, Rybak MJ. The ß-lactams strike back: ceftazidime-avibactam. Pharmacotherapy. 2015;35(8):755-70. https://doi.org/10.1002/phar.1622. 
7. Hidalgo JA, Vinluan CM, Antony N. Ceftazidime/avibactam: a novel cephalosporin/nonbeta-lactam beta-lactamase inhibitor for the treatment of complicated urinary tract infections and complicated intra-abdominal infections. Drug Des Devel Ther. 2016;10:2379-86. 10.2147\%2FDDDT.S110946

8. Lagacé-Wiens P, Walkty A, Karlowsky JA. Ceftazidime-avibactam: an evidence-based review of its pharmacology and potential use in the treatment of gram-negative bacterial infections. Core Evid. 2014;9:13-25. https://doi.org/10.2147/CE.S40698.

9. Barber KE, Ortwine JK, Akins RL. Ceftazidime/avibactam: who says you can't teach an old drug new tricks? J Pharm Pharm Sci. 2016;19(4):448-64. https:// doi.org/10.18433/J3X31R.

10. Capri S, Ceci A, Terranova L, Merlo F, Mantovani L. Guidelines for economic evaluations in Italy: recommendations from the Italian Group of Pharmacoeconomic Studies. Drug Info J. 2001;35:189-201. 10.1177\%2F0092 86150103500122

11. Naylor NR, Atun R, Zhu N, Kulasabanathan K, Silva S, Chatterjee A, et al. Estimating the burden of antimicrobial resistance: a systematic literature review. Antimicrob Resist Infect Control. 2018;7:58. https://doi.org/10.1186/ s13756-018-0336-y.

12. Verhoef TI, Morris S. Cost-effectiveness and pricing of antibacterial drugs. Chem Biol Drug Des. 2015;85(1):4-13. 10.1111\%2Fcbdd.12417

13. Bassetti M, Pecori D, Peghin M. Multidrug-resistant gram-negative bacteriaresistant infections: epidemiology, clinical issues and therapeutic options. Ital J Med. 2016;10(4):364-75. https://doi.org/10.4081/itjm.2016.802.

14. Scarafile G. Antibiotic resistance: current issues and future strategies. Rev Health Care. 2016;7(1):3-16. https://doi.org/10.7175/rhc.v7i1.1226.

15. Liu Q, Li X, Li W, Du X, He JQ, Tao C, et al. Influence of carbapenem resistance on mortality of patients with Pseudomonas aeruginosa infection: a meta-analysis. Sci Rep. 2015;5:11715. https://doi.org/10.1038/srep11715.

16. Marquet K, Liesenborgs A, Bergs J, Vleugels A, Claes N. Incidence and outcome of inappropriate in-hospital empiric antibiotics for severe infection: a systematic review and meta-analysis. Crit Care. 2015;19:63. https://doi.org/ 10.1186/s13054-015-0795-y.

17. Vardakas KZ, Mavroudis AD, Georgiou M, Falagas ME. Intravenous colistin combination antimicrobial treatment vs. monotherapy: a systematic review and meta-analysis. Int J Antimicrob Agents. 2018;51(4):535-47. https://doi. org/10.1016/j.jiantimicag.2017.12.020.

18. Petrosillo N, Giannella M, Lewis R, Viale P. Treatment of carbapenemresistant Klebsiella pneumoniae: the state of the art. Expert Rev Anti-Infect Ther. 2013;11(2):159-77. https://doi.org/10.1586/eri.12.162.

19. Gomez-Simmonds A, Nelson B, Eiras DP, Loo A, Jenkins SG, Whittier S, et al. Combination regimens for treatment of carbapenem-resistant Klebsiella pneumoniae bloodstream infections. Antimicrob Agents Chemother. 2016; 60(6):3601-7. https://doi.org/10.1128/AAC.03007-15.

20. European Centre for Disease Prevention and Control: https://ecdc.europa. eu/sites/portal/files/media/en/publications/Publications/Communicabledisease-threats-report-21-may-2016.pdf. Assessed June 2019

21. Chen Z, Wu J, Zhang Y, et al. Efficacy and safety of tigecycline monotherapy vs imipenem/cilastatin in Chinese patients with complicated intraabdominal infections: a randomized controlled trial. BMC Infect Dis. 2010;10: 217. https://doi.org/10.1186/1471-2334-10-217.

22. Fomin P, Beuran M, Gradauskas A, et al. Tigecycline is efficacious in the treatment of complicated intra-abdominal infections. Int J Surg. 2005;3(1): 35-47. https://doi.org/10.1016/j.ijsu.2005.03.011.

23. Oliva ME, Rekha A, Yellin A, et al. A multicenter trial of the efficacy and safety of tigecycline versus imipenem/cilastatin in patients with complicated intra-abdominal infections [Study ID Numbers: 3074A1-301-WW; ClinicalTrials.gov Identifier: NCT00081744]. BMC Infect Dis. 2005;5:88. https:// doi.org/10.1186/1471-2334-5-88.

24. Qvist N, Warren B, Leister-Tebbe H, Zito ET, Pedersen R, McGovern PC, et al. Efficacy of tigecycline versus ceftriaxone plus metronidazole for the treatment of complicated intra-abdominal infections: results from a randomized, controlled trial. Surg Infect. 2012;13(2):102-9. https://doi.org/10.1089/sur.2011.048

25. Towfigh S, Pasternak J, Poirier A, Leister H, Babinchak T. A multicentre, open-label, randomized comparative study of tigecycline versus ceftriaxone sodium plus metronidazole for the treatment of hospitalized subjects with complicated intra-abdominal infections. Clin Microbiol Infect. 2010;16(8): 1274-81. https://doi.org/10.1111/j.1469-0691.2010.03122x

26. European Medicines Agency: Annex I: summary of product characteristics (Zavicefta). https://www.ema.europa.eu/en/documents/product-information/ zavicefta-epar-product-information_en.pdf. Assessed June 2019
27. European Medicines Agency: Annex I: summary of product characteristics (Doribax). https://www.ema.europa.eu/en/documents/product-information/ doribax-epar-product-information_en.pdf. Assessed June 2019

28. Sturkenboom MC, Goettsch WG, Picelli G, in 't Veld B, Yin DD, de Jong RB, et al. Inappropriate initial treatment of secondary intra-abdominal infections leads to increased risk of clinical failure and costs. Br J Clin Pharmacol. 2005; 60(4):438-43. https://doi.org/10.1111/j.1365-2125.2005.02443.x.

29. Song Y, Tai JH, Bartsch SM, Zimmerman RK, Muder RR, Lee BY. The potential economic value of a Staphylococcus aureus vaccine among hemodialysis patients. Vaccine. 2012;30(24):3675-82. https://doi.org/10.1016/j.vaccine. 2012.03.031.

30. Delate T, Coons SJ. The use of 2 health-related quality-of-life measures in a sample of persons infected with human immunodeficiency virus. Clin Infect Dis. 2001;32:e47-52. https://doi.org/10.1086/318492.

31. Agenzia Italiana del Farmaco (2014) http://www.aifa.gov.it/content/liste-ditrasparenza-e-rimborsabilit\%C3\%A0. Accessed 25 May 2018

32. Ministero della Salute - Direzione generale della programmazione sanitaria. Tariffe delle prestazioni di assistenza ospedaliera per acuti, per tipo di ricovero (Euro) (2009) http://www.salute.gov.it/portale/temi/p2_6. jsp?lingua=italiano\&id=1349\&area=ricoveriOspedalieri\&menu=vuoto. Accessed June 2019

33. Ministero della Salute - Rapporto SDO 2013 - Tavola 2.2.6 - Descrizione dell'attività per DRG (versione 24) - Attività per Acuti in Regime ordinario Anno (2013). http://www.salute.gov.it/portale/documentazione/p6_2_8_3_1. jsp?lingua=italiano\&id=18. Accessed June 2019

34. Tan SS, Bakker J, Hoogendoorn ME, Kapila A, Martin J, Pezzi A, Pittoni G, Spronk PE, Welte R, Hakkaart-van RL. Direct cost analysis of intensive care unit stay in four European countries: applying a standardized costing methodology. Value Health. 2012;15(1):81-6. https://doi.org/10.1016/j.jval. 2011.09.007.

35. Solomkin J, Hershberger E, Miller B, Popejoy M, Friedland I, Steenbergen J, et al. Ceftolozane/tazobactam plus metronidazole for complicated intraabdominal infections in an era of multidrug resistance: results from a randomized, double-blind, phase 3 trial (ASPECT-CIAI). Clin Infect Dis. 2015; 60(10):1462-71. https://doi.org/10.1093/cid/civ097.

36. Committee Joint Formulary. British National Formulary (BNF) 66. Pharmaceutical Press (2013) https://about.medicinescomplete.com/ publication/british-national-formulary/. Accessed 25 May 2018

37. Thitima Kongnakorn, Eszter Tichy, Evie Merinopoulou, Patrizia Berto, Roberto Di Virgilio, Claudie Charbonneau (2017) Budget impact of introducing ceftazidime avibactam (CAZ-AVI) for complicated intra-abdominal infection (CIAI), complicated urinary tract infection (CUTI) and hospital-acquired pneumonia including ventilator-associated pneumonia (HAPNAP) to a hospital formulary in Italy. In: ISPOR $20^{\text {th }}$ Annual European Congress, Glasgow, Scotland, 4-8 November 2017

38. Sartelli M, Catena F, di Saverio S, Ansaloni L, Coccolini F, Tranà C, et al. The challenge of antimicrobial resistance in managing intra-abdominal infections. Surg Infect. 2015;16(3):213-20. https://doi.org/10.1089/sur.2013.262.

39. Barie PS, Rotstein OD, Dellinger EP, Grasela TH, Walawander CA. The costeffectiveness of cefepime plus metronidazole versus imipenem/cilastatin in the treatment of complicated intra-abdominal infection. Surg Infect. 2004; 5(3):269-80. https://doi.org/10.1089/sur.2004.5.269.

40. Giacobbe DR, Mikulska M, Viscoli C. Recent advances in the pharmacological management of infections due to multidrug-resistant Gram-negative bacteria. Expert Rev Clin Pharmacol. 2018;11(12):1219-36. https://doi.org/10. 1080/17512433.2018.1549487.

\section{Publisher's Note}

Springer Nature remains neutral with regard to jurisdictional claims in published maps and institutional affiliations. 